\section{Nr. 34 GOÄ geht öfter, als man denkt}

Nr. 34 GOÄ steht für eine intensive Beratung, wenn bei einem Patienten eine nachhaltig lebensverändernde oder lebensbedrohende Erkrankung festgestellt wurde oder eine solche sich verschlimmert hat. Dabei kann es ggf. auch um die Planung eines operativen Eingriffs gehen. Die Dauer des Gesprächs muss mindestens 20 Minuten betragen. Die Leistung ist im Schwellensatz (2,3fach) mit 40,23 Euro bewertet und kann innerhalb von sechs Monaten höchstens zweimal berechnet werden.

\section{MMW-KOMMENTAR}

Neben der Nr. 34 sind die Nrn. 1, 3, 4, 15 und/ oder 30 in gleicher Sitzung nicht berechnungsfähig. Ebenfalls in gleicher Sitzung

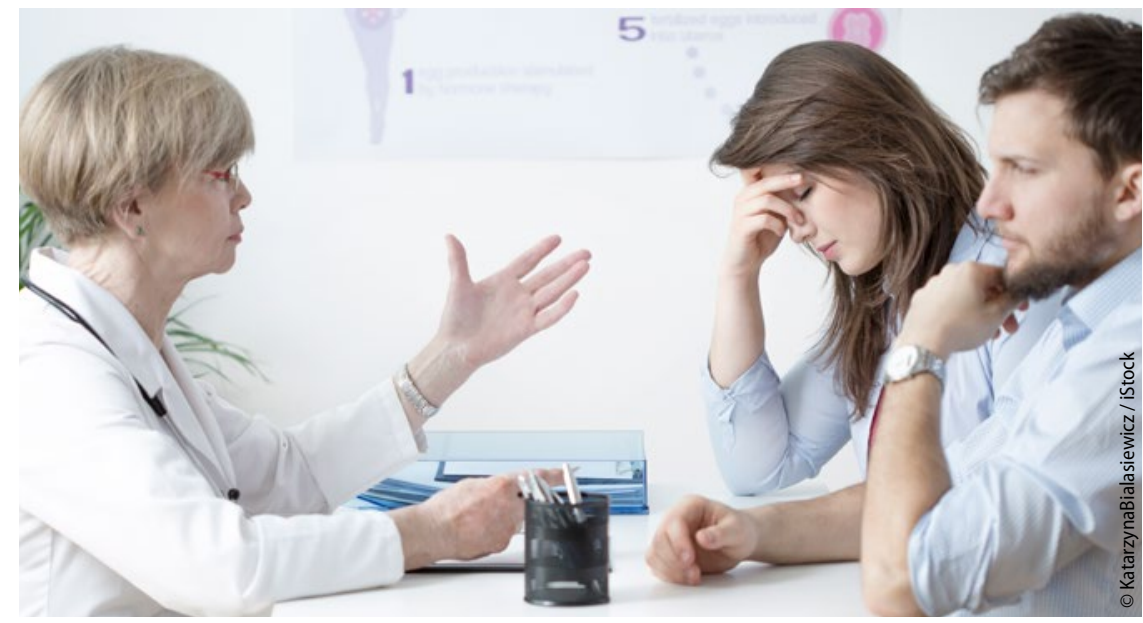

In Zeiten der Krise ist oft eine intensivere ärztliche Beratung nötig.

ausgeschlossen sind die Leistungen nach den Nrn. 2, 21, 22, 30, 31, 435, 804, 806, 807, 808, $812,817,835,849,861,862,863,864,870,871$, 886 und 887. Die Leistung muss persönlich vom Arzt erbracht werden. Dabei ist eine telefonische Beratung wie etwa bei der Nr. 3 nicht möglich. Die Antwort auf die Frage, ob eine Krankheit als „nachhaltig lebensverän- dernd oder lebensbedrohend" einzustufen ist, ergibt sich in der Regel aus dem Gespräch selbst. Das Vorliegen einer chronischen Erkrankung ist dabei nicht gefordert, sodass auch akute Krankheitsfälle durchaus Anlass für den Ansatz der Leistung sein können, wenn die geforderte Gesprächsdauer erfüllt wurde.

\title{
Seltsame EBM-Definition der Chronikerbetreuung
}

_ Im Gegensatz zur eigenständigen $\mathrm{Be}$ treuungsleistung nach Nr. 15 in der GÖ̈ (siehe vorherige Seite) handelt es sich bei den korrespondierenden EBM-Leistungen nach den Nrn. 03220 und 03221 um Zuschläge zu der altersgestaffelten Versichertenpauschale nach Nr. 03000 für die Behandlung und Betreuung eines Patienten mit mindestens einer lebensverändernden chronischen Erkrankung. Obligater Leistungsinhalt ist ein persönlicher Arzt-Patienten-Kontakt. Die Abrechnung ist jeweils im Behandlungsfall und damit in jedem Quartal möglich. Das Vorliegen einer chronischen Erkrankung als weitere Voraussetzung für den Ansatz kann nach der ICD-10Nomenklatur dokumentiert werden.

\section{MMW-KOMMENTAR}

Im Einzelfall ist das Problem beim Ansatz der Nrn. 03220/03221 weniger die Definition der chronischen Erkrankung als vielmehr die völlig realitätsferne Beschreibung einer kontinuierlichen ärztlichen Behandlung im EBM. Diese liegt nur dann vor, wenn in mindestens drei der letzten vier Quartale wegen derselben gesicherten chronischen Erkrankung ein Arzt-Patienten-Kontakt in derselben Praxis stattgefunden hat. In zwei Quartalen muss es auch ein persönlicher Arzt-Patienten-Kontakt gewesen sein. Lediglich bei Neugeborenen und Säuglingen braucht es keine vorherigen Kontakte.

Zu einer Ausnahmesituation kommt es, wenn der chronisch Kranke den Hausarzt wechselt. Der neue Hausarzt muss dann die Arzt-Patienten-Kontakte seines Vorgängers dokumentieren und dies mittels des Zusatzes " $H^{\prime \prime}$ in der Abrechnung kennzeichnen. So wird eine kontinuierliche Betreuung anerkannt.

Konkret bedeutet dies, dass der Patient - sollen die Leistungen in einem Quartal abre- chenbar sein - in den zurückliegenden drei Quartalen nur einmal "gefehlt" haben darf. Zusätzlich muss in mindestens einem der verbleibenden Quartale die Versichertenpauschale abgerechnet worden sein. Beachtenswert ist auch, dass in einem Quartal ohne persönlichen Arzt-Patienten-Kontakt die Leistungen nach den Nrn. 01430/01435 zur Dokumentation des mittelbaren Kontaktes zum Ansatz kommen - und daneben die Chronikerziffern nicht berechnungsfähig sind, da sie ja Zuschläge zur Versichertenpauschale sind. Allein schon aus dem Selbstverständnis einer in der Legende der Nrn. 03220/03221 geforderten „lebensverändernden chronischen" Erkrankung sollte es im hausärztlichen Bereich solche Kontakte aber eigentlich nicht geben - zumal ein einfacher Blickkontakt zwischen Arzt und Patient bereits das Kriterium des „persönlichen Arzt-Patienten-Kontakts" erfüllt. 